\title{
Carbon Nanotube Hybrids for Renewable Energy
}

\section{Levitsky I.A*}

Emitech, Inc., Fall River, Massachusetts and Department of Chemistry, University of Rhode Island, USA

This editorial presents a short summary and perspectives of recent explorations of light energy conversion and related photovoltaic (PV) devices constructed from carbon nanotubes (CNTs) interfaced with organic and inorganic materials. The first reports about CNT photoconductivity [1-3] motivated a growing interest of light energy conversion employing unique optical, electrical, thermal and mechanical properties of carbon nanotubes. Carbon nanotubes as organic, nanoscaled objects [4] outperform their organic counterparts in many aspects, making CNTs the favorite candidate for various optoelectronic applications. For example, semiconducting CNTs have a high light absorbance in visible and near infra-red (NIR) spectrum (absorbance coefficient is in the range of $10^{4}-10^{5} \mathrm{~cm}^{-1}$ ), with a band gap depending on their diameter, while most organic compounds are not capable of absorbing NIR light. Another advantage of CNTs is very high charge mobility (up to $10^{5} \mathrm{~cm}^{2} \mathrm{~V}^{-1} \mathrm{~s}^{-1}$ for individual nanotubes [5] and $\sim 60 \mathrm{~cm}^{2} \mathrm{~V}^{-1} \mathrm{~s}^{-1}$ for CNT films [6]) as compared with conductive organic materials. In addition, carbon nanotubes exhibit an exceptional environmental stability and resistance to photo bleaching which is one of the major drawbacks of organic optoelectronics. Device fabrication is simple and cost effective as CNTs can be easily incorporated in the device's architecture by wet processing (coating, spraying, and printing). Finally, CNTs can be simply doped or functionalized by many covalent and non-covalent routes, forming nano-assemblies with other molecules and polymers to provide an efficient photoinduced charge transfer or tuning Fermi level to the favorable position at heterojunction with semiconductors. CNT light absorption is an initial step leading to a generation of bound excitons. In order to convert light energy into an electrical signal, excitons should be separated on free charge carriers (electrons and holes) by an external or internal built-in electric field, before they relax to the ground state. Finally, the resulting carriers should be transported to the electrodes minimizing the recombination and trapping processes. Such a scenario is realized for photovoltaics and photodetectors when the internal built-in field is required at the interface between carbon nanotubes and other materials. Distinct from pristine CNTs, interface related photoconversion processes are more complex but at the same time very intriguing, rich in novel phenomena and are extremely attractive for many optoelectronics applications. For instance, very recently, a surprisingly high photoconversion efficiency (PCE) of $\sim 14 \%$ for CNT/Si hybrid solar cells has been reported [7], exceeding any PCE for organic and hybrid photovoltaics. Noteworthy, research in the field of CNT/semiconductor PV is very new (just past five years) and limited by few groups [7-10] as compared with substantial efforts and time (about 20 years) spent by PV community in other directions such as polymer based and dye-synthesized solar cells where the best PCE is still in the range of $10-12 \%$ [11-13]. Photoactive CNT/ counterpart nanohybrids include significant diversity in the CNT morphology (network and individual CNTs), their structure (SWNTs and MWNTs) and electronic properties (semiconducting and metallic). The choice of CNT counterpart can also be very different comprising small molecules, oligomers, polymers, quantum dots and semiconductors (bulk and nanostructured). The most studied structures utilized in solar cell design are CNTs/small molecules and CNTs/polymers, where CNTs act as an electron acceptor (with some exceptions) and light is absorbed through the CNT complimentary component. Interestingly, in majority
PV studies of CNT hybrids, the role of CNTs in light harvesting was underestimated in the photocorversion process. Recent reports demonstrated that CNTs can be involved not only in charge separation and transport processes, but also in efficient light absorption [9-14]. It was a great hope in the beginning of $2000^{\text {th }}$ that an incorporation of carbon nanotubes in organic photovoltaic device (OPV) and formation of bulk heterojunction between CNT and polymer or small molecules should significantly improve solar cell performance and boost PCE level beyond of $10 \%$. Nevertheless, despite substantial efforts for almost a decade, the maximum reported PCEs for CNT/polymer devices stay in the range of only $0.2-0.5 \%[15,16]$. Just recent studies $[17,18]$ shed light on exciton dissociation, role of semiconducting and metallic carbon nanotubes and the morphology of single walled carbon nanotube (SWNT) networks. It was demonstrated that the critical role in efficient charge separation and transport in SWNT/polymer heterojunction play semiconducting SWNTs (not metallic) and their debundling minimizing the junction effect. As a rule, the inter-tube junctions in SWNT network is the source of energy barriers and trapping, that substantially reduces the transport of photogenerated carriers. Alternatively, to CNT based OPVs, CNT/semiconductor hybrids opened recently a new horizons in light harvesting for renewable energy sources. Exciting results ( PCE $\sim 14 \%$ ) for CNT/Si PV heterostructure reported last year [7] promise novel achievements in the near future toward stable conversion efficiency exceeding $15 \%$. Especially, CNT can be effectively employed in amorphous $\mathrm{Si}(\mathrm{aSi})$ thin film, flexible solar cells, replacing $p$-type aSi layer. The cost of carbon nanotubes reduces very rapidly every year, while the conversion efficiency of $15 \%$ and higher definitely exceeds any organic/hybrid/aSi $(\sim 8-12 \%)$ thin film devices in the current market. Last but not least, there is an exceptional environmental stability of carbon nanotubes, significantly outperforming any organic compounds. Moreover, the variety of parameters (e.g. CNT chirality, diameter, doping level, Si porosity, etc) allow to further proceed with device optimization leading to $18-20 \%$ PCE level. Cost-effective processing, light weight, flexibility and robustness make these PV devices extremely attractive for various civilian needs using clean technology approach. It is hard to overestimate the benefits of the emergence of a novel and highly competitive CNT nanocomposite based solar cells in the current multibillion dollar market.

\section{References}

1. Fujiwara A, Matsuoka Y, Suematsu H, Ogawa1 N, Miyano K, et al. (2001) Photoconductivity in semiconducting single-walled carbon nanotubes. Jpn J Appl Phys 40: L1229-L1231.

*Corresponding author: Levitsky IA, Emitech, Inc., Fall River, Massachusetts and Department of Chemistry, University of Rhode Island, USA, E-mail: ilevitsky@ emitechinc.com

Received September 18, 2012; Accepted September 20, 2012; Published September 21, 2012

Citation: Levitsky IA (2012) Carbon Nanotube Hybrids for Renewable Energy. J Nanomed Nanotechol 3:e117. doi:10.4172/2157-7439.1000e117

Copyright: @ 2012 Levitsky IA. This is an open-access article distributed under the terms of the Creative Commons Attribution License, which permits unrestricted use, distribution, and reproduction in any medium, provided the original author and source are credited. 
2. Levitsky IA and Euler WB (2003) Photoconductivity of single wall carbon nanotubes under CW NIR illumination. Appl Phys Lett 83: 1857-1859.

3. Freitag M, Martin Y, Misewich JA, Martel R, Avouris PH (2003) Photoconductivity of single carbon nanotubes. Nano Lett 3: 1067-1071.

4. Saito R, Dresselhaus G, Dresselhaus MS (1998) Physical properties of carbon nanotubes. Imperial College Press, London.

5. Dürkop T, Getty SA, Cobas E, Fuhrer MS (2004) Extraordinary mobility in semiconducting carbon nanotubes. Nano Lett 4: 35-39.

6. Xiao K, Liu Y, Hu P, Yu G, Wang X, et al. (2003) High-mobility thin-film transistors based on aligned carbon nanotubes. Appl Phys Lett 83:150-152.

7. Jia Y, Cao A, Bai X, Li Z, Zhang L, et al. (2011) Achieving High Efficiency Silicon-Carbon Nanotube Heterojunction Solar Cells by Acid Doping. Nano Lett 11: $1901-1905$

8. Li Z, Kunets VP, Saini V, Xu Y, Dervishi E, et al. (2009) Light-harvesting using high density $p$-type single wall carbon nanotube/ $n$-type silicon heterojunctions. ACS Nano 3: 1407-1414.

9. Ong PL, Euler WB, Levitsky IA (2010) Hybrid solar cells based on single-walled carbon nanotubes/Si heterojunction. Nanotechnology 21: 105203.

10. Wadhwa P, Liu B, McCarthy MA, Wu Z, Rinzler AG (2010) Electronic Junction Control in a Nanotube-Semiconductor Schottky Junction Solar Cell. Nano Lett 10: $5001-5005$
11. Brabec CJ, Dyakonov V, Scherf U (2008) Organic photovoltaics: materials device physics, and manufacturing technologies. Weinheim : Wiley- $\mathrm{VCH}$.

12. Chen CY, Wang M, Li JY, Pootrakulchote N, Alibabaei L, et al. (2009) Highly efficient light-harvesting ruthenium sensitizer for thin-film dye-sensitized solar cells. ACS Nano 3: 3103-3109.

13. Yu Q, Wang Y, Yi Z, Zu N, Zhang J, et al. (2010) High-efficiency dye-sensitized solar cells: the influence of lithium ions on exciton dissociation, charge recombination, and surface states. ACS Nano 4: 6032-6038.

14. Bindl DJ, Wu MY, Prehn FC, Arnold MS (2011) Efficiently harvesting excitons from electronic type-controlled semiconducting carbon nanotube films. Nano Lett 11: 455-460.

15. Kymakis E, Koudoumas E, Franghiadakis I, Amaratunga GAJ (2006) Postfabrication Annealing Effects in Polymer-Nanotube photovoltaic cells. J Phys D: Appl Phys 39: 1058-1062.

16. Lanzi M, , Paganin L, Caretti D (2008) New photoactive oligo- and Polyalkylthiophenes. Polymer 49: 4942-4948.

17. Holt JM, Ferguson AJ, Kopidakis N, Larsen BA, Bult J, et al. (2010) Prolonging charge separation in P3HT-SWNT composites using highly enriched semiconducting nanotubes. Nano Lett 10: 4627-4633.

18. Ham MH, Paulus GL, Lee CY, Song C, Kalantar-zadeh K, et al. (2010) Evidence for high-efficiency exciton dissociation at polymer/single-walled carbon nanotube interfaces in planar nano-heterojunction photovoltaics. ACS Nano 4: 6251-6259. 